\title{
Research on a Novel Denoising Method of Ultrasonic Measurement Signal
}

\author{
ZHANG Lanyong $^{1 *}, \mathrm{CAO} \mathrm{An}^{1}$ and DU Yixuan ${ }^{1}$ \\ ${ }^{1}$ College of Automation, Harbin Engineering University, Harbin, China \\ zlyalf@sina.com \\ * please mark the corresponding author with an asterisk
}

\begin{abstract}
Keywords: Wavelet threshold denoising, threshold function, ultrasound signal
Abstract. A wavelet threshold denoising method is proposed. The new threshold functions in the method overcome not only the constant deviation between the estimated wavelet coefficients and the decomposition wavelet coefficients in the soft threshold function, but also the discontinuance of the hard threshold function. Compared with several existing threshold functions, the new threshold function is continuous and higher - order derivative. The noise at the boundary of signal can be eliminated by the proposed method. Simulation results confirm that the method is more effective than existing methods in low SNR.
\end{abstract}

\section{Introduction}

In ultrasonic testing systems, ultrasonic echo signal to be received is a time-varying non-stationary signal, and usually mixed with varying degrees of random noise that makes the echo signal distortion, and affect the subsequent signal processing. The traditional de-noising method for stationary signals such as Fourier transform, Wiener filter, etc. are based on the cost of the loss of the useful signal, so how to keep useful signal as much as possible while removing the noise became popular areas of current research ${ }^{[1]}$. The wavelet transform has good time-frequency domain localized nature, so the wavelet technology has been widely studied in the signal de-noising and made a very good application effects.

Donoho proposed wavelet threshold denoising method is one of the most commonly used wavelet de-noising methods ${ }^{[2]}$. Finding the suitable threshold and threshold processing structure is the key of wavelet threshold denoising method. The commonly used threshold processing function is the hard threshold and soft threshold function proposed by Donoho ${ }^{[3]}$. Where in the hard threshold function is discontinuous at the threshold, the processed signal will produce additional shocks. Although the soft threshold function is continuous at the threshold value, but greater than the threshold value of wavelet coefficients of wavelet coefficients and wavelet coefficients exist a fixed deviation which will cause the loss of the useful signal. Some methods have been proposed some improvements for the shortcomings of hard and soft threshold function ${ }^{[4]}$. The threshold processing function proposed in document ${ }^{[4]}$ is the compromise of soft and hard threshold, but is still not a good solution to the discontinuity problem of threshold function. The threshold function proposed in the literature ${ }^{[3]}$ is continuous at the threshold and have higher order derivatives of rectifiable, but in the case of low SNR, eliminating noise performance is poor. This paper presents a new threshold handler function, it is continuity and has higher order derivatives of rectifiable at the threshold value, can also retain the original information of the signal. At the same time, the noise can be more effectively removed and the noise signal at the boundary. The simulation results show that the improved wavelet threshold de-noising method has better performance than conventional methods.

\section{The principles and methods of Wavelet threshold de-noising method.}

Signal Model. Assuming a one-dimensional signal model with noise is ${ }^{[5]}$ :

$$
X(n)=f(n)+\sigma * e(n)
$$


Wherein $X_{(n)}$ is the observed signal. $f(n)$ is the true signal, $\sigma$ is the noise variance, $e(n)$ is Gaussian white noise, normally distributed. The real purpose of de-noising is to extract the true signal from the noise pollution signals.

Wavelet Threshold De-noising Theoretical Basis. Orthogonal wavelet transform has a strong correlation to the data, it can make the signal energy concentrated in some large coefficients in the wavelet domain; the noise energy is distributed throughout the wavelet domain, therefore, after the wavelet decomposition, wavelet coefficients amplitude of the signal will be greater than the amplitude of the noise coefficient. Take it that the relatively large amplitude of the wavelet coefficient are generally based on the signal, and a relatively small amplitude is the noise in large. So using the threshold approach could reserve signal coefficient, leaving most of the noise factor reducing to zero. In fact, it is already proved in the mean square error sense threshold method to get the best approximation estimate of the original signal.

The Steps of Wavelet Threshold De-noising Method. Wavelet threshold de-noising method has three main steps ${ }^{[7]}$ :

Step One: choose an appropriate wavelet and determine the wavelet decomposition number of layers N. Then signal $X(n)$ can be regarded as $\mathrm{N}$ layer of wavelet decomposition. We can get a group of wavelet coefficients, including the low-frequency coefficients $a_{N, k}$ of the $\mathrm{N}$-scale, and high-frequency coefficients of various scales in the $j=1,2 \mathrm{~L} N . \mathrm{k}$ is the sampling point number. Step two: as high-frequency coefficients for each layer from the first layer to the layer $\mathrm{N}$, select a threshold and threshold processing functions quantify the threshold. Then we can obtain the wavelet coefficients $\hat{d_{j, k}}$ after treatment. There are four commonly used threshold selection method: fixed threshold(sqtwolog), using stein unbiased likelihood estimation principle of threshold selection(rigrsure), heuristic threshold selection(heursure) and mini-max threshold(mini-maxi).

The selected the threshold processing functions are usually soft threshold function, hard threshold and a variety of processing functions

Step three: according to the low-frequency coefficients of wavelet decomposition of the N-layer and high-frequency after quantized coefficients of the first layer to the N-layer wavelet, perform wavelet reconstruction of one-dimensional signal. We can get the signal which noise has been removed

Select the threshold processing function. Soft Threshold and Hard Threshold. The commonly used threshold processing functions are hard threshold and soft threshold proposed by Donoho handler which hard threshold function expression is ${ }^{[8]}$ :

$$
\hat{d_{j, k}}= \begin{cases}d_{j, k} & \hat{d_{j, k}>t h r} \\ 0 & \hat{d_{j, k}>t h r}\end{cases}
$$

When the wavelet coefficients are greater than the threshold $t h r$, hard threshold function has not changed for wavelet coefficients, and therefore retains the better high-frequency signal. However, due to the hard threshold function discontinuity at the threshold, it will cause greater added shocks in the reconstructed signal, and affect the smoothness of the signal.

Soft threshold function:

$$
\hat{d_{j, k}}= \begin{cases}\operatorname{sign}\left(d_{j, k}\right)\left(\left|d_{j, k}\right|-t h r\right) & \hat{d_{j, k}}>t h r \\ 0 & \hat{d_{j, k}}>t h r\end{cases}
$$

Where $\operatorname{sign}(9$ is the sign function, Soft threshold function is continuous at the threshold value thr, so the resulting reconstructed signal has a good smoothness. However, because the processing wavelet coefficients and the original wavelet coefficients exist constant differences when the wavelet 
coefficients are greater than the threshold value in the interval, the signal loss some high-frequency information.

Several Improved Threshold Handler. Document ${ }^{[4]}$ proposes a compromise soft and hard threshold function:

$$
\hat{d_{j, k}}= \begin{cases}\operatorname{sign}\left(d_{j, k}\right)\left(\left|d_{j, k}\right|-\alpha * t h r\right) & \hat{d_{j, k}}>t h r \\ 0 & \hat{d_{j, k}}>t h r\end{cases}
$$

This handler function is a compromise of soft and hard threshold, when $\alpha=0$. The function reduces to a soft threshold function, when $\alpha=1$. The function reduces to a hard threshold function. When $0<\alpha<1$, its denoising performance is better than hard and soft threshold function. However, this function is continuous at the threshold only when $\alpha=0$, and has no higher derivative.

The literature ${ }^{[3]}$ proposes esp. type threshold function which has a continuity higher order derivatives. Its expression is:

$$
\hat{d}_{j, k}= \begin{cases}\operatorname{sign}\left(d_{j, k}\right)\left[\left|d_{j, k}\right|-T h r / \exp \left(\frac{\left|d_{j, k}\right|-t h r}{N}\right)\right] & \left|d_{j, k}\right|>t h r \\ 0 & \left|d_{j, k}\right| \leq t h r\end{cases}
$$

When $N \rightarrow \infty$, this quantify function reduces to the hard threshold handler. When $N \rightarrow 0$, this quantify function reduces to the soft threshold handler. This handler at the threshold can be continuous and have higher-order derivative. However, because of the increasing of $C_{j, k}$, exp function increases rapidly, $\hat{C_{i, k}}$ is also fast approaching $\hat{C_{j, k}}$. In the case of small signal to noise ratio, the size difference between the wavelet coefficients of the wavelet coefficients of the noise and the signal was not very clear. We have reasons to believe that possibility of wavelet coefficients at the boundary of the threshold with noise is larger. Therefore, the performance of such a threshold function to the noise at the boundary of the threshold is not very satisfactory.

A New Wavelet Threshold Function. Threshold wavelet functions analyses above have been widely applied in practice, but still have their drawbacks. To overcome these shortcomings, this paper proposes a new threshold handler. Its expression is:

$$
\hat{d}_{j, k}= \begin{cases}\operatorname{sign}\left(d_{j, k}\right)\left[\left|d_{j, k}\right|-t h r / L n\left(\frac{\left|d_{j, k}\right|-t h r+N^{*} e}{N}\right)\right] & \left|d_{j, k}\right|>t h r \\ 0 & \left|d_{j, k}\right| \leq t h r\end{cases}
$$

Wherein $\mathrm{N}$ is a positive number greater than zero, $t h r$ is taken as a positive integer multiple. Now examine the limit type:

$$
\lim _{\left|d_{j, k}\right| \rightarrow \infty} \frac{\hat{d}_{j, k}}{d_{j, k}}=\lim _{\left|d_{j, k}\right| \rightarrow \infty} \operatorname{sign}\left(d_{j, k}\right)\left[1-t h r /\left|d_{j, k}\right| \operatorname{Ln}\left(\frac{\left|d_{j, k}\right|-t h r+N^{*} e}{N}\right)\right]=\operatorname{sign}\left(d_{j, k}\right)
$$

Visible, when $d_{j, k}$ tends to infinity, $\hat{d_{j, k}}$ tends to $d_{j, k}$. Further investigate the effect of $\mathrm{N}$ on the threshold function by examining the following two limit formula:

$$
\begin{aligned}
\lim _{N \rightarrow \infty} \hat{d_{j, k}} & =\lim _{N \rightarrow \infty} \operatorname{sgn}\left(d_{j, k}\right)\left[\left|d_{j, k}\right|-t h r / \operatorname{Ln}\left(\frac{d_{j, k} \mid-t h r+N^{*} e}{N}\right)\right] \\
& =\operatorname{sgn}\left(d_{j, k}\right)\left(\left|d_{j, k}\right|-t h r\right) \quad\left|d_{j, k}\right|>t h r
\end{aligned}
$$




$$
\begin{aligned}
\lim _{N \rightarrow 0} \hat{\hat{d}_{j, k}} & =\lim _{N \rightarrow 0} \operatorname{sgn}\left(d_{j, k}\right)\left[\left|d_{j, k}\right|-t h r / \operatorname{Ln}\left(\frac{\left|d_{j, k}\right|-t h r+N^{*} e}{N}\right)\right] \\
& =d_{j, k} \quad\left|d_{j, k}\right|>t h r
\end{aligned}
$$

This shows that when $N \rightarrow \infty$, new threshold function tends to be soft threshold function, and when $N \rightarrow 0$, the new threshold function tends to be hard threshold function. Therefore, the new threshold is a compromise of soft and hard threshold, while the threshold function also has the continuity at the threshold and higher-order derivative at $\left|d_{j, k}\right|>t h r$. Compared to formula (5), the speed that $\hat{d_{j, k}}$ tends to $d_{j, k}$ is slower. If the threshold was set smaller, the wavelet coefficients which near the threshold were more likely affected by noise control. Then the new threshold function will be more conducive to eliminate the noise around the threshold value which is in the boundary of noise and the signal. Ultrasonic echo signals typically have a lower signal to noise ratio. Since typically useful information usually embedded in high-frequency components, the use of threshold is more conservative. Therefore, the threshold processing functions proposed for de-noising ultrasonic echo signal in this paper has better adaptability.

Contrast the new threshold function and soft and hard threshold and threshold function Exp type shown in Figure 1.

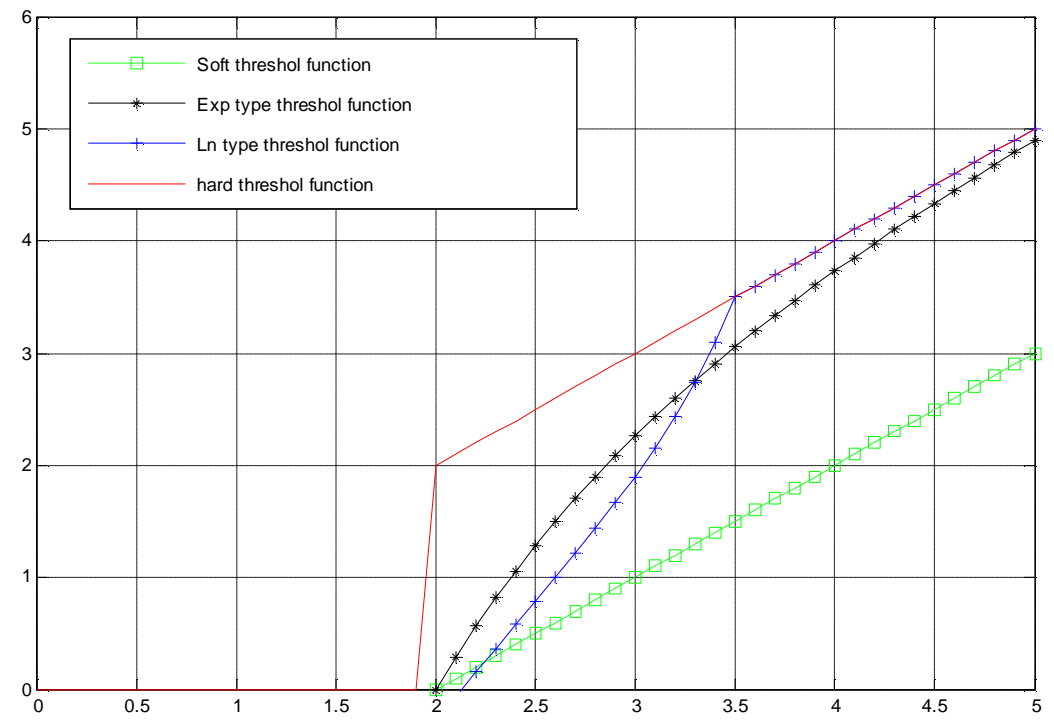

Figure 1 Comparison of the various of the threshold function

\section{Experimental verification.}

In order to verify the effectiveness of the new threshold function, use the heavy sine signal in Matlab which is more like to ultrasonic echo signal to do simulation. In this experiment we use sym7's type、 5 decomposition level the max-min threshold rule and 10dB SNR. Renderings after the simulation are shown in Figure 2. 

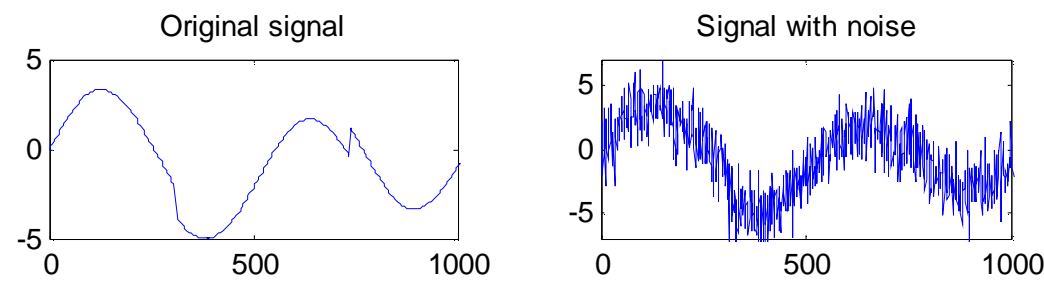

Signal after hard threshold de-noising

Signal after Ln threshold de-noising
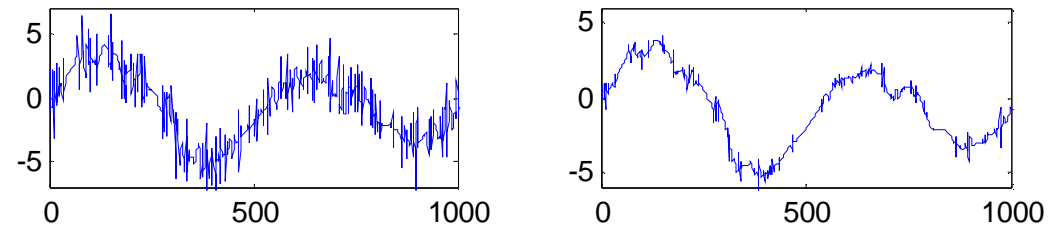

Signal after Exp threshold de-noising

Signal after soft threshold de-noising
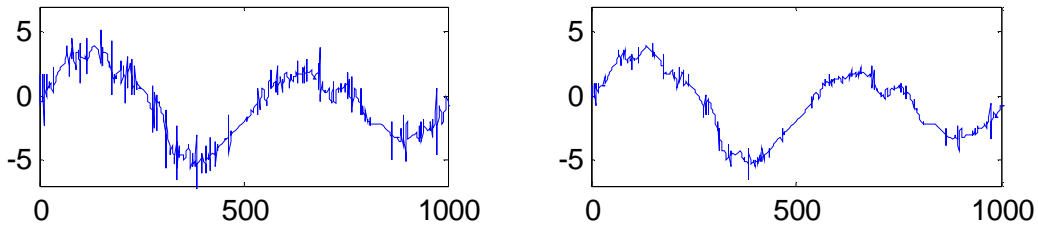

Figure 2 Signal in a variety threshold function of denoising effect

To compare the denoising effect of various threshold, calculated their mean square error RMSE and after de-noising SNR, which are shown in Table 1.

Table 1 SNR and RMSE of signal at various threshold function

\begin{tabular}{|l|l|l|}
\hline & SNR & RMSE \\
\hline Hard threshold function & 18.7834 & 0.4786 \\
\hline Soft threshold function & 23.5339 & 0.3766 \\
\hline Exp type threshold function & 24.2167 & 0.3219 \\
\hline Ln type threshold function & 25.2147 & 0.2872 \\
\hline
\end{tabular}

Under different SNR, the curve of signal in Exp and the new Ln type threshold handler proposed in this paper is shown in Figure 3.

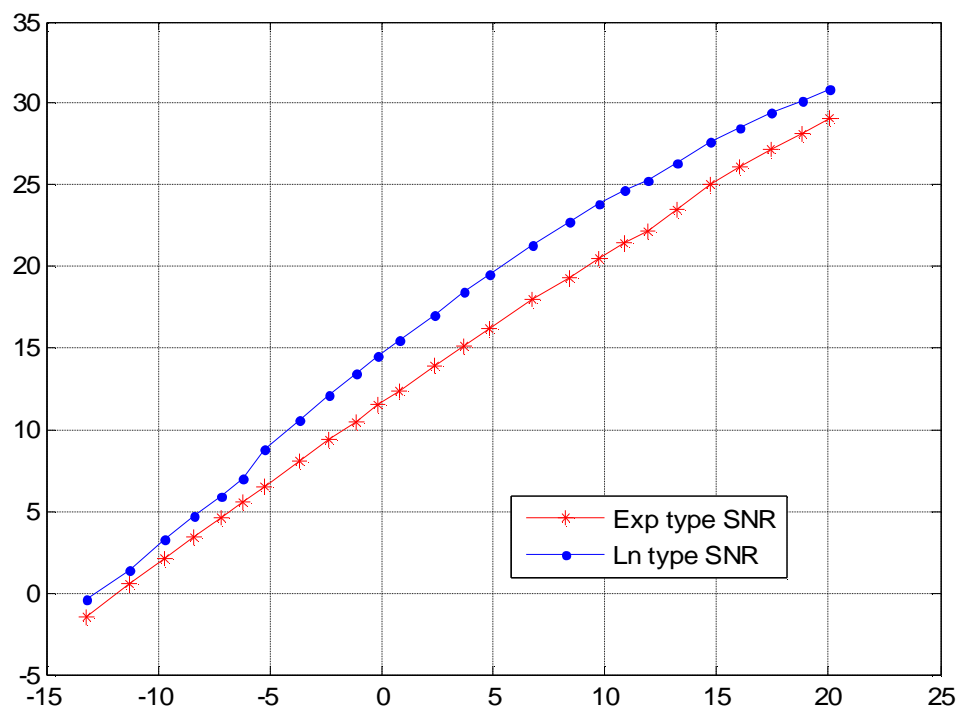

Figure 3: Comparison with two different SNR thresholding

Figure 1 and Table 1 verify that the hard threshold processing function after noising exist large shocks. Soft threshold processing functions after the noise cancellations are smoothed. However, it 
attenuated the high frequency information. Exp improved threshold and the new Ln type threshold handler proposed in this paper in both smoothness and accuracy are better. Picture 3 verified that in low SNR, Ln type threshold function is better than the proposed threshold Exp type Handler de-noising. The reason is that in the case of low SNR, wavelet coefficients near the threshold value was greater probability generated by the noise, and Ln type threshold processing functions can weaken wavelet coefficients of the threshold

\section{Conclusions.}

Aiming at the shortcomings of hard and soft threshold function and some existing improved thresholds handler, this paper proposed a new threshold processing function. Through the simulation of various signal, it can obtained that it can effectively remove the noise and the noise signal at the boundary. When in low SNR, it has better de-noising effect and adaptability for the ultrasonic echo signal. It has a high value in engineering.

\section{Acknowledgment.}

This work was supported in part by Research Fund for the Doctoral Program of Higher Education 20132304120015, Doctoral Scientific Research Foundation of Heilongjiang under No. LBH-Q14040, Heilongjiang Government Postdoctoral Foundation under No.LBH-12078 and Fundamental Research Funds for the Central Universities under HEUCFX41305.

\section{References}

[1] Soszynska, Joanna. Reliability and risk evaluation of a port oil pipeline transportation system in variable operation conditions[J]. International Journal of Pressure Vessels and Piping, 2010, $177(2-3)$, pp.81-87

[2] Koornneef, Joris, et al. Quantitative risk assessment of $\mathrm{CO} 2$ transport by pipelines-A review of uncertainties and their impacts[J]. Journal of Hazardous Materials, 2010, 177(1-3) , pp.2-27.

[3] Martins, Jaqueline Costa, et al. Assessment of the performance of acoustic and mass balance methods for leak detection in pipelines for transporting liquids [J]. Journal of Fluids Engineering, 2010, 132(1), pp.0114011-0114018.

[4] Tatar O., Cirebea C., Alutei A., Mândru D.. The design of adaptable indoor pipeline inspection robots. 2010 IEEE International Conference on Automation, Quality and Testing, Robotics, 2010, 1, pp.325-328.

[5] Dong Yongsheng, Yi Xu ming. Wavelet de-noising based on four improved functions for threshold estimation[J]. Journal of Mathematics, 2006,26(5), pp.473-477.

[6] Tao G,MaX1,Ling Y.Optimal and nonlinear decoupling control of systems with sandwiched backlash[J].Automatic,2001,37(2),pp.165-176.

[7] Johnstone I M. Adapting to unknown smoothness via wavelet shrinkage[J]. Journal of American Statistic Association, 2003,12(90), pp.684-692.

[8] GAO Hong-Ye, BRUCE A G. WaveShrink with firm shrinkage[J]. Statistica Sinica, 2004,7(4): 855-874.

[9] Hasan, Shadi W, et al. Heavy crude oil viscosity reduction and rheology for pipeline transportation[J]. Fuel, 2010, 89(5), pp.1095-1100

[10] Dong Yongsheng, Yi Xu ming. Wavelet de-noising based on four improved functions for threshold estimation[J]. Journal of Mathematics, 2006, 26(5), pp.473-477. 